\title{
Universitäre Forschung und private Innovation
}

\section{Brigitte Tschudi}

\section{Relevanz}

Mit ihren Kernaufgaben in Forschung und Lehre ermöglichen die Universitäten Wachstum durch Innovation. Sie bilden die Studierenden an der Grenze der Forschung aus und versorgen die Privatwirtschaft mit international konkurrenzfähigem F\&E-Personal. In der Grundlagenforschung erarbeiten sie neues Wissen und legen den Grundstein für viele mögliche Anwendungen und Innovationen. Ein steter Wissenstransfer von den Universitäten in den privaten Sektor ist jedoch bei weitem nicht selbstverständlich. Die Forscher müssen Zeit und großen Aufwand tätigen, um neue Anwendungen zu erschließen und Patente zu entwickeln. Diese Investitionen fehlen dann wieder in der Lehre und Grundlagenforschung. Damit die Ergebnisse der universitären Forschung für private Innovationen nutzbar werden, muss sich der Aufwand lohnen. Gute Rahmenbedingungen für den Wissenstransfer müssen daher Anreize für die Forscher setzen, Aufwand in die Entwicklung neuer Anwendungen und Patente zu lenken.

Christian Keuschnigg

\section{Quelle}

Der nachfolgende Text ist eine Zusammenfassung von: Hvide, Hans K. und Benjamin F. Jones (2016), University Innovation and the Professor's Privilege, NBER Working Paper No. 22.057, 1-66.

B. Tschudi $(\bowtie)$

Universität St. Gallen, St. Gallen, Schweiz

E-Mail: brigitte.tschudi@student.unisg.ch

(C) Der/die Autor(en) 2018

C. Keuschnigg (Hrsg.), Inklusives Wachstum und wirtschaftliche Sicherheit, https://doi.org/10.1007/978-3-658-21344-2_14 
Innovationen schieben die technologische Grenze hinaus, steigern die Produktivität und sind ein mächtiger Treiber des Wirtschaftswachstums. Eine bedeutende Rolle im Innovationsprozess spielen die Universitäten. Die dort beschäftigten Wissenschaftler schaffen durch ihre Forschungsarbeit neues Wissen. Sie transferieren dieses Wissen in die Wirtschaft, indem sie neues F\&E-Personal ausbilden, Start-ups gründen oder mit Lizenzen und Patenten mithelfen, die Erkenntnisse der Grundlagenforschung für private Innovationen zu erschließen und zu kommerzialisieren. Universitäten sind die Geburtsstätte zahlreicher innovativer Ideen. Aufgrund der zentralen Rolle der Innovation für das Wachstum haben Forschungs- und Technologiepolitik in den meisten Ländern höchste Priorität auf der wirtschaftspolitischen Agenda. Regierungen versuchen mit verschiedenen Maßnahmen, die Forschung im Allgemeinen und den Technologietransfer von den Universitäten in die Privatwirtschaft im Speziellen zu fördern.

Die Autoren untersuchen in ihrer Studie eine spezielle Maßnahme, die den Technologietransfer stark beeinflussen kann. Konkret analysieren sie, wie sich in Norwegen die Abschaffung der sogenannten „Professorenbeteiligung“ an den finanziellen Erträgen der universitären Forschung („Professor's Privilege“) im Jahr 2003 auf die Anzahl und die Qualität von Startups und von Patenten universitärer Forscher ausgewirkt hat. Aufgrund dieses Privilegs genossen Forscher, die an Universitäten angestellt waren, umfassende Rechte an den von ihnen geschaffenen Innovationen und insbesondere an den Einkünften aus Start-ups und Patenten. Mit der Abschaffung der Professorenbeteiligung wurden diese Rechte jedoch an die Universitäten übertragen, die nun zwei Drittel der Nettoeinnahmen behalten dürfen. Bislang hatten Forscher die gesamten Einnahmen erhalten. Mit einer stärkeren Beteiligung der Universitäten an den Forschungserlösen sollte die Reform die Kommerzialisierung der universitären Forschung fördern. Die empirische Evidenz zeigt jedoch das genaue Gegenteil.

Norwegen hat 2003 die Beteiligung der Wissenschaftler an den finanziellen Erträgen ihrer Forschung abgeschafft und die Einnahmen den Universitäten übertragen.

Die Autoren nutzen für ihre Studie einen umfassenden Datensatz über neu geschaffene Firmen und Patente sowie deren Gründer bzw. Erfinder. Von den 48.844 Start-ups, die im Zeitraum zwischen 2000 und 2007 in Norwegen gegründet wurden, stammten 128 aus den Universitäten. Universitäre Start-ups zeichnen 
sich durch eine höhere Überlebenswahrscheinlichkeit aus, weisen allerdings weniger Mitarbeitende sowie einen geringeren Umsatz und Gewinn auf. Zudem konnten zwischen 1995 und 2010750 Patentanmeldungen durch 431 universitäre Forschende verzeichnet werden. Im Vergleich dazu wurden in ganz Norwegen 7341 Patente von 6890 Erfindern angemeldet.

Bereits die beschreibende Statistik lässt auf einen negativen Zusammenhang zwischen der Abschaffung der Professorenbeteiligung und der Anzahl von Start-ups schließen. Während die Zahl der neugegründeten, nicht-universitären Start-ups im Untersuchungszeitraum ungefähr konstant blieb, ist ein erheblicher Rückgang bei universitären Neugründungen auszumachen. So sank die Zahl der universitären Start-ups von durchschnittlich 24,7 vor der Reform (2000-2002) auf durchschnittlich 10,8 universitäre Start-ups pro Jahr danach (2003-2007). Dies entspricht einem Rückgang von $56 \%$. Dieser negative Zusammenhang wird durch verschiedene Regressionen bestätigt. Die Autoren schätzen beispielsweise, dass die Anzahl der Start-ups, welche universitätsangehörige Forscher (PhD-Absolventen) im Durchschnitt gründen, infolge der Reform zwischen $49 \%$ und $67 \%$ sinkt. Zwar sind diese Resultate auf Basis aggregierter Daten mit Vorsicht zu interpretieren, da sie auf nur 16 Beobachtungen beruhen, jedoch zeigen individuelle Daten, welche dagegen in sehr großer Zahl vorliegen, ähnliche Ergebnisse. So sinkt die Wahrscheinlichkeit, dass ein universitätsangehöriger Forscher ein Unternehmen gründet, um rund $63 \%$.

Die Zahl universitärer Neugründungen sank von durchschnittlich 24,7 pro Jahr vor der Reform auf 10,8 danach. Das ist ein Rückgang von $56 \%$. Die Reform senkt die Wahrscheinlichkeit, dass ein universitärer Forscher ein Unternehmen gründet, um ca. 63 \%.

Der erwähnte Rückgang bei den universitären Neugründungen könnte zwei Gründe haben. Forscher, die ihre Innovationen kommerzialisieren, könnten die Universitäten häufiger verlassen, so dass ihre Gründungen nicht mehr als universitär zählen, oder die an den Universitäten verbleibenden Forscher könnten weniger kommerzialisierbare Innovationen schaffen. Die Autoren zeigen, dass nicht der Weggang der Forscher für den beobachteten Rückgang verantwortlich gemacht werden kann, sondern die geringere Neigung an den Universitäten, Zeit und Aufwand in die Kommerzialisierung der Forschung zu stecken. Zudem kommen die Wissenschaftler zum Schluss, dass die Reform auch die Qualität der 
Neugründungen beeinträchtigte. So sank z. B. die Überlebenswahrscheinlichkeit universitärer Start-ups um 15 Prozentpunkte. Ein statistisch signifikanter Rückgang konnte zudem bei deren Umsatz beobachtet werden. Schließlich gingen die universitären Neugründungen im Hightech-Bereich deutlich zurück.

Ähnliche Ergebnisse zeigen sich auch bei der Zahl der Patente an den Universitäten. Im Vergleich zu den nicht-universitären Patentanmeldungen gehen die gesamten Patentanmeldungen einer Universität um 20 und jene eines universitätsangehörigen Forschers um $48 \%$ zurück. Dies geht mit einer Verringerung der Wahrscheinlichkeit einher, dass ein Forscher an einer Universität ein Patent entwickelt. Die Abschaffung der Professorenbeteiligung reduziert diese Wahrscheinlichkeit um 4,5 Prozentpunkte. Wie vorhin konnten die Autoren nachweisen, dass dieser Rückgang nicht darauf zurückgeht, dass besonders aktive Forschende ihre Universitätsanstellung aufgegeben haben. Der Grund liegt vielmehr darin, dass die an den Universitäten verbleibenden Forscher nach der Reform wesentlich seltener Patente entwickelten. Die ungünstige Anreizwirkung der Reform mindert auch die Qualität der universitären Patente, gemessen an der Anzahl ihrer Zitationen. Die Häufigkeit, dass ein Patent genannt wird, geht um rund $25 \%$ zurück, was einer durchschnittlichen Abnahme von 2,2 Nennungen entspricht.

Nach der Abschaffung des Professorenanteils gingen die gesamten Patentanmeldungen einer Universität um $20 \%$ und jene eines universitären Forschers um $48 \%$ zurück.

Die empirischen Ergebnisse deuten darauf hin, dass die Abschaffung der Professorenbeteiligung in Norwegen das genaue Gegenteil des beabsichtigten Effektes zur Folge hatte. Das Ausmass der Kommerzialisierung sowie deren Qualität sanken beträchtlich. Finanzielle Anreize sollten nicht bei der Universität als Ganzes, sondern bei den einzelnen Forschern ansetzen, die Zeit und Aufwand von ihren anderen Aufgaben in Forschung und Lehre abzweigen und in die Kommerzialisierung ihrer Forschungsergebnisse stecken müssen.

Die Ergebnisse stützen auch die Idee, dass Eigentumsrechte für die Entstehung von Innovationen bedeutend sind. Oft wird angenommen, dass Wissenschaftler in einer universitären Umgebung sich weniger von rein finanziellen Anreizen leiten lassen und eine größere Bereitschaft an den Tag legen, die Früchte ihrer Arbeit mit der Öffentlichkeit zu teilen. Aber auch an den Universitäten bringt eine 
Schwächung der Eigentumsrechte eine starke Abnahme der davon betroffenen Forschungsaktivitäten mit sich. Wer also mehr Technologietransfer haben will, muss das Interesse der in Frage kommenden Forscher wecken, kommerzialisierbare Anwendungen zu erschließen, und sie am Erfolg dieser Aktivitäten beteiligen.

Open Access Dieses Kapitel wird unter der Creative Commons Namensnennung 4.0 International Lizenz (http://creativecommons.org/licenses/by/4.0/deed.de) veröffentlicht, welche die Nutzung, Vervielfältigung, Bearbeitung, Verbreitung und Wiedergabe in jeglichem Medium und Format erlaubt, sofern Sie den/die ursprünglichen Autor(en) und die Quelle ordnungsgemäß nennen, einen Link zur Creative Commons Lizenz beifügen und angeben, ob Änderungen vorgenommen wurden.

Die in diesem Kapitel enthaltenen Bilder und sonstiges Drittmaterial unterliegen ebenfalls der genannten Creative Commons Lizenz, sofern sich aus der Abbildungslegende nichts anderes ergibt. Sofern das betreffende Material nicht unter der genannten Creative Commons Lizenz steht und die betreffende Handlung nicht nach gesetzlichen Vorschriften erlaubt ist, ist für die oben aufgeführten Weiterverwendungen des Materials die Einwilligung des jeweiligen Rechteinhabers einzuholen.

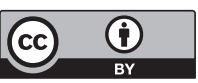

